\title{
Appendix
}

\section{Survey Items}

1. In the next two weeks, how likely are you to do each of the following in response to concerns about coronavirus?

\begin{tabular}{|c|c|c|c|c|c|c|c|}
\hline & $\begin{array}{c}\text { Extremely } \\
\text { unlikely } \\
1\end{array}$ & 2 & 3 & $\begin{array}{c}\text { Neutral } \\
4\end{array}$ & 5 & 6 & $\begin{array}{c}\text { Extremely } \\
\text { likely } \\
7\end{array}$ \\
\hline $\begin{array}{l}\text { Avoid people } \\
\text { who look } \\
\text { Asian on } \\
\text { public } \\
\text { transportation } \\
\text { like buses } \\
\text { and trains }\end{array}$ & 0 & & & C & & & \\
\hline
\end{tabular}

2. SUSCEPT_1 It is likely that I will get coronavirus

Strongly disagree 1

Disagree 2

Neither agree nor disagree 3

Agree 4

Strongly agree 5 
3. SUSCEPT_2 It is likely that someone in my family will get coronavirus

Strongly disagree 1

Disagree 2

Neither agree nor disagree 3

Agree 4

Strongly agree 5

4. SUSCEPT_3 It is likely that someone in my community will get coronavirus

Strongly disagree 1

Disagree 2

Neither agree nor disagree 3

Agree 4

Strongly agree 5

5. SEVERE_1 If I were to get the coronavirus, it would be a very serious threat to my health.

Strongly agree 1

2

Neither agree nor disagree 3

4

Strongly disagree 5 
6. SEVERE_2 If I were to get the coronavirus, it would be a very serious threat to members of my family.

Strongly agree 1

2

Neither agree nor disagree 3

4

Strongly disagree 5

7. SEVERE_3 If I were to get the coronavirus, it would be a very serious threat to members of my community.

Strongly agree 1

2

Neither agree nor disagree 3

4

Strongly disagree 5

8. How much do you agree with each of the following statements? 


\begin{tabular}{|c|c|c|c|c|c|c|c|}
\hline & $\begin{array}{c}\text { Strongly } \\
\text { disagree } \\
1\end{array}$ & 2 & 3 & $\begin{array}{c}\text { Neither } \\
\text { agree nor } \\
\text { disagree } \\
4\end{array}$ & 5 & 6 & $\begin{array}{c}\text { Strongly } \\
\text { agree } \\
7\end{array}$ \\
\hline $\begin{array}{l}\text { Asian people are } \\
\text { more likely to } \\
\text { carry the virus } \\
\text { than other } \\
\text { people. }\end{array}$ & & & & & & & \\
\hline $\begin{array}{c}\text { Eating at Chinese } \\
\text { restaurants } \\
\text { increases your } \\
\text { risk of } \\
\text { coronavirus more } \\
\text { than eating at } \\
\text { other restaurants. }\end{array}$ & & & & & & & \\
\hline
\end{tabular}

9. Now we are going to ask you a few questions about how you feel in certain situations. Please indicate how much you agree or disagree with the following statements about you: 
Strongly

disagree

1
2

Neither agree

nor disagree

3
Strongly

4 agree

5

\section{I don't like}

situations that are uncertain.

It's frustrating to listen to someone who cannot make up his or her mind.

I dislike questions which could be answered in many different ways

I do not usually consult many different options before forming my own view.

When considering most conflict situations, I can usually see how both sides could be right. 
10. Please rate how DISGUSTING you find the following

\begin{tabular}{|c|c|c|c|c|c|c|c|}
\hline & $\begin{array}{c}\text { Not at all } \\
\text { disgusting } \\
1\end{array}$ & 2 & 3 & $\begin{array}{c}\text { Somewhat } \\
\text { disgusting } \\
4\end{array}$ & 5 & 6 & $\begin{array}{c}\text { Extremely } \\
\text { disgusting } \\
7\end{array}$ \\
\hline $\begin{array}{l}\text { Seeing some } \\
\text { mold on old } \\
\text { leftovers in } \\
\text { your } \\
\text { refrigerator }\end{array}$ & 0 & 0 & 0 & 0 & $\bigcirc$ & $\bigcirc$ & 0 \\
\hline $\begin{array}{l}\text { Standing } \\
\text { close to a } \\
\text { person who } \\
\text { has body } \\
\text { odor }\end{array}$ & 0 & $\bigcirc$ & 0 & 0 & 0 & 0 & 0 \\
\hline $\begin{array}{c}\text { Shaking } \\
\text { hands with a } \\
\text { stranger } \\
\text { who has } \\
\text { sweaty } \\
\text { palms }\end{array}$ & 0 & $\bigcirc$ & 0 & 0 & $\bigcirc$ & $\bigcirc$ & 0 \\
\hline $\begin{array}{l}\text { Accidentally } \\
\text { touching a } \\
\text { person's } \\
\text { bloody cut }\end{array}$ & 0 & 0 & 0 & 0 & $\bigcirc$ & $\bigcirc$ & 0 \\
\hline $\begin{array}{l}\text { Seeing a } \\
\text { cockroach } \\
\text { run across } \\
\text { the floor }\end{array}$ & 0 & 0 & 0 & 0 & $\bigcirc$ & 0 & 0 \\
\hline $\begin{array}{l}\text { Sitting next } \\
\text { to someone } \\
\text { who has red } \\
\text { sores on } \\
\text { their arm }\end{array}$ & 0 & 0 & 0 & 0 & $\bigcirc$ & 0 & 0 \\
\hline
\end{tabular}


11. Please describe how much time you spend doing each below:

I do not

do this at

all

1
I do this

2

3 sometimes

4
I do this

6

a lot

7

\footnotetext{
I actively

search news

media to help

me

understand

about the

coronavirus.

I ask my

close friends

and family

members

about the

coronavirus.

I follow

social media

to acquire

information

about the

coronavirus.

I engage in conversations with others about the coronavirus.
} 


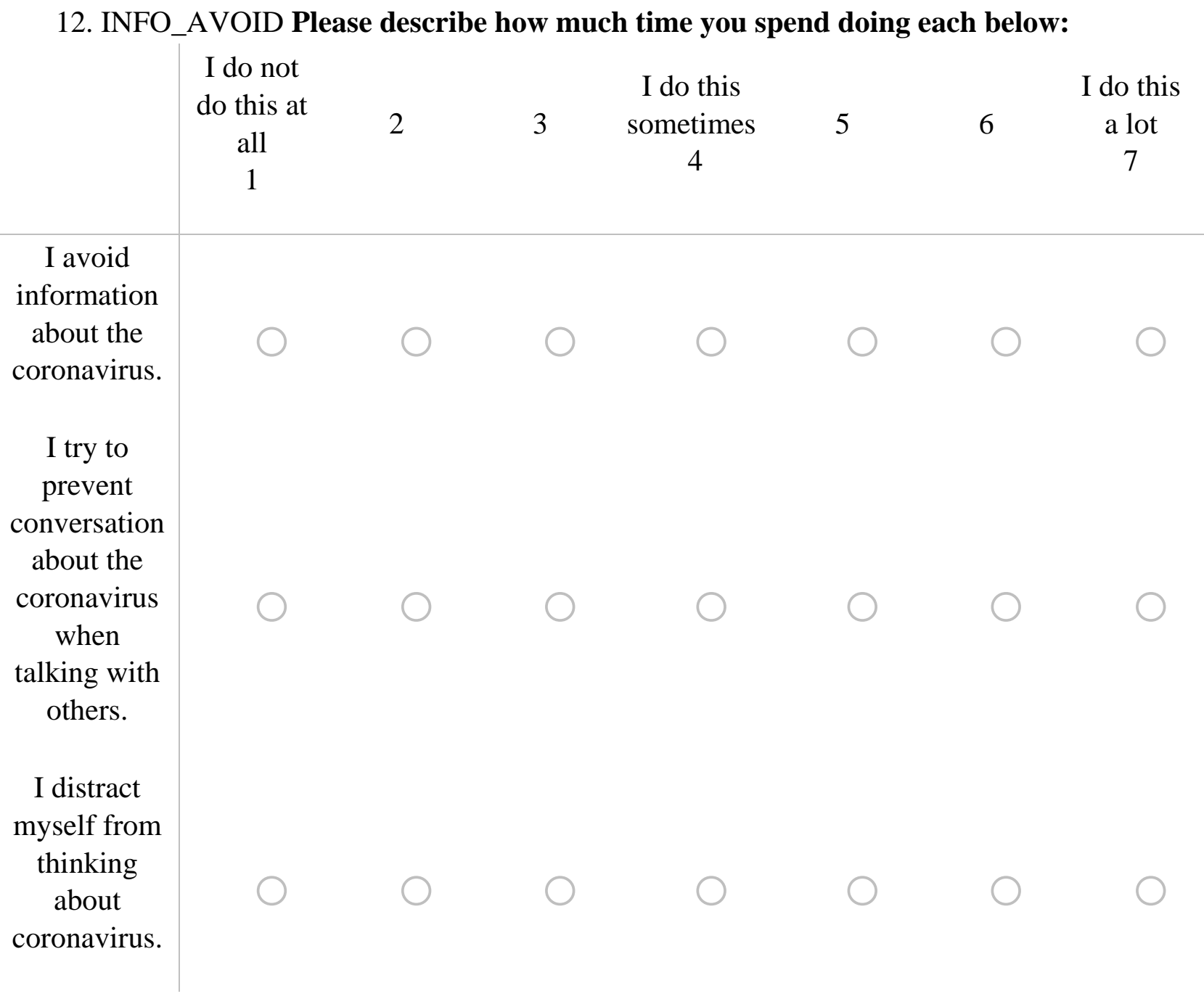

13. Please rate the following public officials on a 0-100 feeling thermometer where $\mathbf{0}$ means EXTREMELY UNFAVORABLE and 100 means EXTREMELY FAVORABLE.

$\begin{array}{llllllllllll}0 & 10 & 20 & 30 & 40 & 50 & 60 & 70 & 80 & 90 & 100\end{array}$

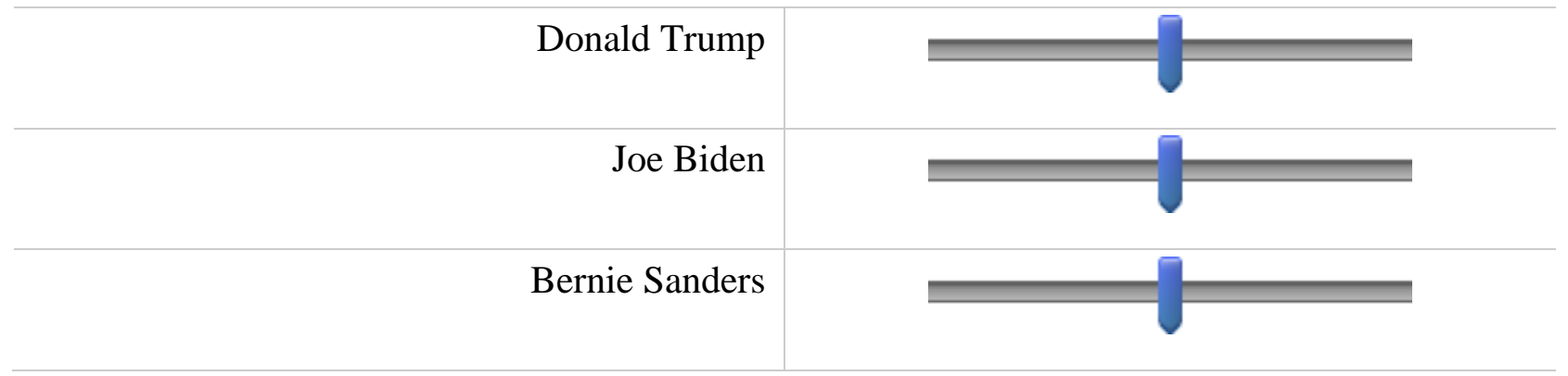


14. UNDERSTANDABLE How acceptable do you think these behaviors are in response to the coronavirus?

\begin{tabular}{|c|c|c|c|c|c|c|c|}
\hline & $\begin{array}{c}\text { Acceptable } \\
1\end{array}$ & 2 & 3 & 4 & 5 & 6 & $\begin{array}{c}\text { Unacceptable } \\
7\end{array}$ \\
\hline $\begin{array}{c}\text { Making } \\
\text { jokes about } \\
\text { Asians, like } \\
\text { calling the } \\
\text { coronavirus } \\
\text { the "Kung } \\
\text { Flu." }\end{array}$ & 0 & 0 & 0 & $\bigcirc$ & 0 & 0 & 0 \\
\hline $\begin{array}{l}\text { Telling } \\
\text { Asian } \\
\text { people in } \\
\text { public that } \\
\text { the } \\
\text { coronavirus } \\
\text { is their } \\
\text { fault. }\end{array}$ & 0 & $\bigcirc$ & 0 & 0 & 0 & 0 & 0 \\
\hline $\begin{array}{l}\text { Physically } \\
\text { attacking } \\
\text { Asians who } \\
\text { are wearing } \\
\text { masks to } \\
\text { get them to } \\
\text { leave } \\
\text { where you } \\
\text { are. }\end{array}$ & 0 & 0 & 0 & 0 & 0 & 0 & 0 \\
\hline $\begin{array}{l}\text { Calling the } \\
\text { coronavirus } \\
\text { the } \\
\text { "Chinese } \\
\text { virus" }\end{array}$ & 0 & 0 & 0 & 0 & 0 & 0 & 0 \\
\hline
\end{tabular}

15. IDEOLOGY In general, would you consider your political views as...

Very liberal 
Liberal

Somewhat liberal

Moderate

Somewhat conservative sc

Conservative

Very conservative

Other

16. GENDER Which of the following best describes your gender identity?

Male

Female

17. What is the highest level of school you have completed or the highest degree you have received?

Less than high school (Grades 1-8 or no formal schooling)

High school incomplete (Grades 9-11 or Grade 12 with NO diploma)

High school graduate (Grade 12 with diploma or GED certificate or vocational, business, technical or other training that did not count toward a degree)

Some college, no degree (includes some community college)

Two year associate degree from a college or university

Four year college or university degree/Bachelor's degree (e.g., BS, BA, AB)

Some postgraduate or professional schooling, no postgraduate degree

Postgraduate or professional degree, including master's, doctorate, medical or law degree (e.g., MA, MS, PhD, MD, JD) 
